\title{
MIXED AUTOMORPHIC FORMS AND DIFFERENTIAL EQUATIONS
}

\author{
MIN HO LEE \\ Department of Mathematics \\ University of Northern Iowa \\ Cedar Falls, Iowa 50614 \\ (Received August 29, 1989 and in revised form April 26, 1990)
}

ABSTRACT. We construct mixed automorphic forms associated to a certain class of nonhomogeneous linear ordinary differential equations. We also establish an isomorphism between the space of mixed automorphic forms of the second kind modulo exact forms and a certain parabolic cohomology explicitly in terms of the periods of mixed automorphic forms.

KEY WORDS AND PHRASES. Mixed automorphic forms, differentia1 equations, parabolic cohomology.

1980 AMS SUBJECT CLASSIFICATION CODES. 11F12, $11 \mathrm{~F} 75$.

\section{INTRODUCTION.}

In this paper we sha11 consider mixed automorphic forms associated to certain differential equations and obtain results similar to the ones in [8].

In [8] P. Stiller considered a certain class of linear ordinary differential equations with regular singular points on a smooth algebraic curve $X$. To each nonhomogeneous differential equation determined by a homogeneous linear differential equation and an element $Z$ in the function field $K(X)$ of $X$ he associated a generalized automorphic form of the second kind. As a generalized Eichler-Shimura isomorphism he established an isomorphism between generalized automorphic forms of the second kind modulo exact forms and a certain parabolic cohomology group. He determined the isomorphism explicitly in terms of the periods of the automorphic forms.

Mixed automorphic forms arise naturally as holomorphic forms on elliptic varieties (see $\S 1$ of this paper, [2], [3], [4] and [5] for details) and they include classical automorphic forms as a special case. The main purpose of this paper is to associate a mixed automorphic form to each nonhomogeneous differential equation determined by an element $Z$ in $K(X)$ and establish an isomorphism similar to the one in [8] described above. We shall also extend the Stiller's explicit description of the isomorphism for classical automorphic forms to the case of mixed automorphic forms. To be more precise, we shall determine the isomorphism in terms of the periods of mixed automorphic forms.

I would like to thank the referee for various helpful suggestions. 
1. MIXED AU'TOMORPHIC FORMS

Let $\Gamma_{1} \subset \operatorname{PSL}(2, R)$ be a torsion free Fuchsian group of the first kind. Let $\left(i: \Gamma_{1} \rightarrow \operatorname{SL}(2, Z)\right.$ be a homomorphism such that $\Gamma_{2}=\alpha\left(\Gamma_{1}\right)$ has finite index in $\mathrm{SL}(2, \mathrm{Z})$. Then both $\mathrm{I}_{1}$ and $\Gamma_{2}$ act on the Poincaré upper half plane $H$ by linear transformations. Let $\mathrm{v}: \mathrm{H} \rightarrow \mathrm{H}$ be a holomorphic map such that

$$
v(\gamma z)=\alpha(\gamma) v(z) \text { for all } \gamma \in \Gamma_{1} \text { and } z \in H \text {. }
$$

We shall assume that the inverse images of parabolic subgroups of $\Gamma_{2}$ are parabolic subgroups of $\Gamma_{1}$ and that the $\Gamma_{1}$-cusps map to $\Gamma_{2}$-cusps.

DEFINITION 1.1. Let $k, m$ be nonnegative integers with $k$ even. A mixed automorphic form of type $(k, m)$ is a meromorphic function $\mathrm{f}: \mathrm{H} \rightarrow \mathrm{C}$ which satisfies the following conditions:

(i) $\quad f(\gamma z)=\left(c_{1} z+d_{1}\right)^{k}\left(c_{2} v(z)+d_{2}\right)^{m} f(z)$,

where

$$
\gamma=\left(\begin{array}{ll}
a_{1} & b_{1} \\
c_{1} & d_{1}
\end{array}\right) \quad \epsilon \Gamma_{1}, \alpha(\gamma)=\left(\begin{array}{ll}
a_{2} & b_{2} \\
c_{2} & d_{2}
\end{array}\right) \in \Gamma_{2} .
$$

(ii) $f$ is meromorphic at the $\Gamma_{1}$-cusps.

In the above definition if $f$ is holomorphic and $f$ vanishes at the $\Gamma_{1}-$ cusps, then $f$ is called a mixed cusp form of type (k,m) (see [2], [3], [4], [5]). We denote by $s_{k, m}\left(\Gamma_{1}, v,(t)\right.$ the set of all mixed cusp forms of type $(k, m)$ associated to $\Gamma_{1}, \mathrm{v}, x$.

Let $X=\Gamma_{1} \backslash H \cup\left\{\Gamma_{1}\right.$-cusps $\}$. Suppose that $\alpha: \Gamma_{1} \rightarrow \operatorname{SL}(2, Z)$ is the monodromy representation for an elliptic fibration $\pi: \mathrm{E} \rightarrow \mathrm{X}$ and that $\mathrm{v}: \mathrm{H} \rightarrow \mathrm{H}$ is the lifting of the period map (see [2], [3], [4] for details). Let $E^{m}$ be the elliptic variety of dimension $\mathrm{m}+1$ associated to the elliptic fibration $\pi: \mathrm{E} \rightarrow \mathrm{X}$ (see [3], [7]).

PROPOSITION 1.2. There is a canonical isomorphism

$$
\mathrm{S}_{2, \mathrm{~m}}\left(\Gamma_{1}, \mathrm{v}, \alpha\right) \cong \mathrm{H}^{0}\left(\mathrm{E}^{\mathrm{m}}, \Omega^{\mathrm{m}+1}\right) \text {. }
$$

PROOF. See [3, Theorem 3.2].

2. DIFFERENTIAL EQUATIONS AND MONODROMY.

In this section we shall describe some of the results given in [8]. Let $x$ be a proper smooth connected curve over $C$. Consider an $n-t h$ order linear homogeneous differential equation on $\mathrm{X}$

$$
\Lambda^{n} f=\frac{d^{n} f}{d x^{n}}+P_{n-1} \frac{d^{n-1} f}{d x}+\ldots+P_{1} \frac{d f}{d x}+P_{0} f=0,
$$

where $x$ is a nonconstant element of the function field $K(X)$ of $X$ and $P_{i}$ 's are in $K(X)$ for $i=1, \ldots, n-1$. We shall assume that $\Lambda^{n}$ has regular singular points.

Let $U \subset X$ be a Zariski open set on which the functions $P_{i}$ are all regular and also $x$ is a good local parameter. Let $\omega_{1}, \ldots, \omega_{n}$ be holomorphic solutions of $\Lambda^{\mathrm{n}} \mathrm{f}=0$ which form a basis for the space of solutions near a fixed point $\mathrm{x}_{0} \in U$. By analytic continuation of the solutions $\omega_{1}, \ldots, \omega_{n}$ around each closed path $\gamma \in \pi_{1}\left(\mathrm{U}, \mathrm{x}_{0}\right)$, we obtain a representation $\rho: \pi_{1}\left(\mathrm{U}, \mathrm{x}_{0}\right) \rightarrow \mathrm{GL}(2, \mathrm{C})$ called a monodromy representation. 
For $Z \in K(X)$ consider the nonhomogeneous equation $\Lambda^{n} f=\%$. If necessary, shrink the Zariski open set $U$ so that \% is also regular on $U$. The solutions of this equation are given by

$$
f=\sum_{i=1}^{n}\left(\int_{x_{0}}^{x} \frac{(-1)^{i+n_{\hat{A}_{i} Z}}}{w} d x+c_{i}\right) \omega_{i}
$$

where $W$ is the Wronskian of the solutions $\omega_{1}, \ldots, \omega_{n}, \widehat{A}_{1}$ is the determinant obtained from $W$ by deleting the $i-t h$ row and the $n$-th column, and $c_{i} \in C$ is a constant. Suppose that the analytic continuation of a solution $f$ around a closed path $\gamma \in \pi_{1}\left(U, x_{0}\right)$ is

$$
f+a_{1} w_{1}+\ldots+a_{n} w_{n} .
$$

The vector $a_{\gamma}=\left(a_{1}, \ldots, a_{n}\right)$ is called the period of $f$ around $\gamma$. If $\gamma$, $T \epsilon$ $\pi_{1}\left(U, x_{0}\right)$ and $M_{\gamma}=\rho(\tau) \in G L(n, C)$, then we have

$$
a_{\gamma \tau}=a_{\tau}+a_{\gamma} M_{\tau}
$$

hence the vector $a_{\gamma}$ determines a cohomology class in the group cohomology

$$
H^{1}\left(\pi,\left(U, x_{0}\right), v_{x_{0}}\right)
$$

with respect to the monodromy representation. Here $v_{x_{0}}$ denotes the space of local solutions of $\Lambda^{\mathrm{n}} \mathrm{f}=0$ at $\mathrm{x}_{0} \in \mathrm{U}$.

THEOREM 2.1. The cohomology class in

$$
\mathrm{H}^{1}\left(\pi_{1}\left(\mathrm{U}, \mathrm{x}_{0}\right), \mathrm{V}_{\mathrm{x}_{0}}\right)
$$

determined by $Z \in K(X)$ is trivial if and only if $Z$ is exact, i.e., if and only if there is $Z^{\prime} \in K(X)$ such that $\Lambda^{n} Z^{\prime}=Z$.

PROOF. See [8, Theorem 3.16].

Let $S \subset X$ be the set of singular points of $\Lambda^{n}$ with nontrivial local monodromy and let $x_{0}=x-s$. We denote by $\operatorname{LE}\left(\Lambda^{n}\right)$ the set of elements $Z \in K(x)$ that are locally exact on $x$. Let

$$
\mathrm{H}_{\mathrm{LE}}^{1}\left(\pi_{1}\left(\mathrm{x}_{0}, \mathrm{x}_{0}\right), \mathrm{v}_{\mathrm{x}_{0}}\right)
$$

be the subgroup of

$$
\mathrm{H}^{1}\left(\pi_{1}\left(\mathrm{x}_{0}, \mathrm{x}_{0}\right), \mathrm{v}_{\mathrm{x}_{0}}\right)
$$

consisting of the cocycles corresponding to the locally exact elements $Z \in K(X)$. Thus by Theorem 2.1 we have

$$
H_{L E}^{1}\left(\pi_{1}\left(x_{0}, x_{0}\right), v_{x_{0}}\right) \cong L E\left(\Lambda^{n}\right) /\left\{\Lambda^{n_{2}} \cdot \mid Z^{\prime} \in K(X)\right\} .
$$

3. MIXED AUTOMORPHIC FORMS ASSOCIATED TO DIFFERENTIAL EQUATIONS.

Let $X$ be a proper smooth connected curve over $C$. As in $\S 2$, we set $x_{0}=$ $X-S$, where $S \subset X$ is the set of singular points of $\Lambda^{n}$ with nontrivial monodromy. Consider a second order linear differential equation

$$
\Lambda^{2} f=\frac{d^{2} f}{d x^{2}}+P_{1}(x) \frac{d f}{d x}+P_{0}(x) f=0
$$

where $x \in K(X)$ is nonconstant and $P_{0}, P_{1} \in K(X)$. If $\omega_{1}, \omega_{2}$ are linearly 
independent solutions of $\Lambda^{2} f=0$, let $s^{m}\left(\Lambda^{2}\right) f=0$ be the $(m+1)$-st order differential equation whose linearly independent solutions are

$$
\omega_{1}^{m}, \omega_{1}^{m-1} \omega_{2}, \ldots, \omega_{1} \omega_{2}^{m-1}, \omega_{2}^{m} \text {. }
$$

Then these solutions form a basis of the solution space of the equation $s^{\mathrm{m}}\left(\Lambda^{2}\right) \mathrm{f}=0$ and the monodromy representation for this equation is

$$
i^{\mathrm{m}} \circ \rho_{\Lambda^{2}, \omega_{1}, \omega_{2}}
$$

where

$$
\rho_{\Lambda}^{2}, \omega_{1}, \omega_{2}: \pi_{1}\left(X_{0}, x_{0}\right) \rightarrow G L(2, C)
$$

is the monodromy representation for $\Lambda^{2}$ and $\Sigma^{m}: G L(2, C) \rightarrow G L(m, C)$ is the $m-t h$ symmetric power homomorphism.

We shall assume that the universal cover of $x_{0}$ is the Poincaré upper half plane $H$ and that $x_{0}=\Gamma \backslash H$ with $\Gamma=\pi_{1}\left(X_{0}, x_{0}\right) \subset \operatorname{SL}(2, Z)$. We shall also assume that

$$
\rho_{\Lambda}{ }^{2}, \omega_{1}, \omega_{2}(\Gamma) \subset \operatorname{SL}(2, R) \text {. }
$$

Let $\pi: H \rightarrow X_{0}$ be the projection map and choose $z_{0} \in$ H such that $\pi\left(z_{0}\right)=x_{0}$. If $f$ is the general solution of the equation $s^{m}\left(\Lambda^{2}\right) f=Z$ with $Z \in K(X)$, then we can pull the functions $\omega_{1}, \omega_{2}$ and $f$ back via $\pi$ to functions near $z_{0}$ which we denote by $\omega_{1}(z), \omega_{2}(z)$ and $f(z)$. We can consider these functions as meromorphic functions on $\mathrm{H}$ via meromorphic continuations. We set

$$
F(z)=\frac{f(z)}{\omega_{2}(z)^{m}}=\sum_{i=1}^{m+1}\left(\int_{x_{0}}^{x} \frac{(-1)^{i+m+1} \hat{A}_{i} z}{W_{S} m\left(\Lambda^{2}\right)}\left(\frac{d x}{d z}\right)^{m+1} d z+c_{i}\right)^{2} \omega(z)^{m+1-i}
$$

(see $[8$, p. 32]).

Now we shall determine a mixed automorphic form of type $(2, \mathrm{~m})$ associated to a solution $f$ to the differential equation

$$
\mathrm{s}^{\mathrm{m}}\left(\Lambda^{2}\right) \mathrm{f}=\mathrm{Z}, \quad \mathrm{Z} \in \operatorname{LE}\left(\mathrm{S}^{\mathrm{m}}\left(\Lambda^{2}\right)\right) .
$$

As an analogy with Eichler [1] and Stiller [8, p.32], we set $g(z)=\frac{d}{d z}\left(\frac{d^{m} F}{d \omega^{m}}\right)$.

PROPOSITION 3.1. We have

$$
F(z)=\frac{1}{m !} \int_{z_{0}}^{z} g(t)(\omega(z)-\omega(t))^{m} d t+\sum_{i=1}^{m+1} c_{i} \omega(z)^{m+1-i}
$$

for some constants $c_{i} \in C$.

PROOF. By integration by parts we have

$$
\begin{array}{r}
\int_{z_{0}}^{z} \frac{d}{d t}\left(\frac{d^{m} F(t)}{d \omega(t)^{m}}\right)(\omega(z)-\omega(t))^{m} d t=-\left(\omega(z)-\omega\left(z_{0}\right)\right)^{m} \frac{d^{m} F}{d \omega^{m}}\left(z_{0}\right) \\
+m \int_{z_{0}}^{z}(\omega(z)-\omega(t))^{m-1} \frac{d^{m} F(t)}{d \omega(t)^{m}} d \omega(t) .
\end{array}
$$

Applying integration by parts $\mathrm{m}-1$ times, the right hand side of the above equation becomes 


$$
\begin{gathered}
-\left(\omega(z)-\omega\left(z_{0}\right)\right)^{m} \frac{d^{m} F}{d \omega^{m}}\left(z_{0}\right)+(-1)^{2} m\left(\omega(z)-\omega\left(z_{0}\right)\right)^{m-1} \frac{d^{m-1} F}{d \omega^{m-1}}\left(z_{0}\right) \\
+(-1)^{3} m(m-1)\left(\omega(z)-\omega\left(z_{0}\right)\right)^{m-2} \frac{d^{m-2} F}{d \omega^{m-2}}\left(z_{0}\right)+\ldots \\
\ldots+(-1)^{m}{ }_{m}(m-1) \ldots 2\left(\omega(z)-\omega\left(z_{0}\right)\right) \frac{d F}{d \omega}\left(z_{0}\right)+m ! \int_{z_{0}}^{z^{m}} \frac{d F(t)}{d \omega(t)} d \omega(t) .
\end{gathered}
$$

Hence we have

$$
\int_{z_{0}}^{z} g(t)(\omega(z)-\omega(t))^{m} d \omega(t)=\sum_{i=1}^{m+1} \tilde{c}_{i} \omega(z)^{m+1-i}+m ! F(z)
$$

for $\tilde{c}_{i} \in C$. Thus the proposition follows.

PROPOSITION 3.2. $g(z)$ is a mixed automorphic form of type $(2, \mathrm{~m})$ associated to $\Gamma, \omega$ and $\rho_{\Lambda}{ }^{2}, \omega_{1}, \omega_{2} \cdot$

PROOF. Since $d^{m+1} F(z) / d(z)^{m+1}$ is a generalized automorphic form of weight $m+2$ (see $[8])$, we have

$$
\begin{aligned}
\frac{d}{d(\gamma z)}\left(\frac{d^{m} F(\gamma z)}{d \omega(\gamma z)^{m}}\right) & =\frac{d^{m+1} F(\gamma z)}{d \omega(\gamma z)^{m+1}} \frac{d \omega(\gamma z)}{d(\gamma z)} \\
& =\left(c_{1} \omega(z)+d_{1}\right)^{m+2} \frac{d^{m+1} F(z)}{d \omega(z)^{m+1}}\left(c_{1} \omega(z)+d_{1}\right)^{-2}(c z+d)^{2} \frac{d \omega(z)}{d z} \\
& =(c z+d)^{2}\left(c_{1} \omega(z)+d_{1}\right)^{m} \frac{d}{d z}\left(\frac{d^{m} F(z)}{d \omega(z)^{m}}\right),
\end{aligned}
$$

where

$$
\gamma=\left(\begin{array}{ll}
a & b \\
c & d
\end{array}\right) \in \Gamma \text { and } \rho_{\Lambda}^{2}, \omega_{1}, \omega_{2}(\gamma)=\left(\begin{array}{ll}
a_{1} & b_{1} \\
c_{1} & d_{1}
\end{array}\right) \in \operatorname{SL}(2, Z) \text {. }
$$

Thus $g(z)$ is a mixed automorphic form of type $(2, \mathrm{~m})$ associated to

$$
\Gamma, \omega \text { and } \rho_{\Lambda}{ }^{2}, \omega_{1}, \omega_{2} \cdot
$$

DEFINITION 3.3. If $Z \in \operatorname{LE}\left(S^{\mathrm{m}}\left(\Lambda^{2}\right)\right)$, the mixed automorphic form $g(z)$ of type $(2, \mathrm{~m})$ determined by $\mathrm{s}^{\mathrm{m}}\left(\Lambda^{2}\right) \mathrm{f}=\mathrm{Z}$ is said to be of the second $k$ ind. Furthermore, if $Z=s^{m}\left(\Lambda^{2}\right) Z^{\prime}$ for some $Z^{\prime} \in K(X)$, then $g(z)$ is said to be exact.

Thus the cohomology group

$$
\mathrm{H}_{\mathrm{LE}}^{1}\left(\Gamma, \mathrm{v}_{\mathrm{s}}^{\mathrm{m}}\left(\Lambda^{2}\right), \mathrm{x}_{0}\right)
$$

can be described as the mixed automorphic forms of type $(2, \mathrm{~m})$ of the second kind modulo exact forms.

4. PERIODS OF MIXED AUTOMORPHIC FORMS.

In the previous section we associated a mixed automorphic form $g(z)$ of type $(2, m)$ to each differential equation of the form $s^{m}\left(\Lambda^{2}\right) f=z, \quad z \in \operatorname{LE}\left(s^{m}\left(\Lambda^{2}\right)\right)$. In this section we shall express the cocycles in 


$$
\mathrm{H}_{\mathrm{LE}}^{\mathrm{l}}\left(\Gamma, \mathrm{v}_{\mathrm{S}}^{\mathrm{m}}\left(\Lambda^{2}\right), \mathrm{x}_{0}\right)
$$

corresponding to mixed automorphic forms of the second kind in terms of the periods of mixed automorphic forms.

Let $f$ be a solution to the differential equation

$$
S^{m}\left(\Lambda^{2}\right) f=Z, \quad Z \in \operatorname{LE}\left(S^{m}\left(\Lambda^{2}\right)\right) \text {. }
$$

If the analytic continuation of $f$ around $\gamma \in \Gamma=\pi_{1}\left(x_{0}, x_{0}\right)$ is

$$
\mathrm{f}+\mathrm{a}_{1} \omega_{1}^{\mathrm{m}}+\ldots+\mathrm{a}_{\mathrm{m}+1} \omega_{2}^{\mathrm{m}} \text {, }
$$

then the vector $a_{\gamma}=\left(a_{1}, \ldots, a_{m+1}\right)$ is a cocycle in

$$
\mathrm{H}_{\mathrm{LE}}^{1}\left(\Gamma, \mathrm{v}_{\mathrm{S}}^{\mathrm{m}}\left(\Lambda^{2}\right), \mathrm{x}_{0}\right)
$$

(see §2). Let $\mathrm{P}_{\mathrm{m}}$ be the $(\mathrm{m}+1) \times(\mathrm{m}+1)$ matrix defined by

$$
(u z-v w)^{m}=\left(u^{m}, u^{m-1} v, \ldots, v^{m}\right) P_{m}^{t}\left(w^{m}, w^{m-1} z, \ldots, z^{m}\right) .
$$

If $\Sigma^{\mathrm{m}}: \mathrm{SL}(2, \mathrm{C}) \rightarrow \mathrm{SL}(\mathrm{m}+\mathrm{l}, \mathrm{C})$ is the $\mathrm{m}$-th symmetric power homomorphism, then we have

$$
{ }^{\mathrm{t}} \Sigma^{\mathrm{m}}(\sigma) \mathrm{P}_{\mathrm{m}} \Sigma^{\mathrm{m}}(\sigma)=\mathrm{P}_{\mathrm{m}}, \quad \mathrm{t}_{\mathrm{P}_{\mathrm{m}}}=(-1)^{\mathrm{m}_{\mathrm{P}}} \mathrm{m}_{\mathrm{m}}
$$

for each $\sigma \in \operatorname{SL}(2, C)$. If $a_{\gamma}$ is a cocycle in $H_{L E}^{1}\left(\Gamma, v_{S} m\left(\Lambda^{2}\right), x_{0}\right)$, we set

$$
a_{\gamma}^{p}=m ! M^{m} P_{m}^{-1} t_{\gamma}
$$

where $M_{\gamma}^{m}$ is the m-th symmetric power of the $(2 \times 2)$-matrix $M_{\gamma}$ (see $\left.[8, p .46]\right)$. Then we have

$$
a_{\gamma \tau}^{P}=a_{\gamma}^{P}+M_{\gamma}^{m} a_{\tau}^{P} \text { for } \gamma, \tau \in \Gamma
$$

hence $a_{\gamma}^{P}$ is a cocycle in the parabolic cohomology group $H_{P}^{1}\left(\Gamma, v_{S} m\left(\Lambda^{2}\right), x_{0}\right)$ of Eichler and Shimura $([1],[6])$.

PROPOSITION 4.1. The assignment $\left\{a_{\gamma}\right\} \mapsto\left\{a_{\gamma}^{P}\right\}$ determines an isomorphism

$$
\mathrm{H}_{\mathrm{LE}}^{1}\left(\Gamma, \mathrm{v}_{\mathrm{S}}^{\mathrm{m}}\left(\Lambda^{2}\right), \mathrm{x}_{0}\right) \cong \mathrm{H}_{\mathrm{P}}^{1}\left(\Gamma, \mathrm{v}_{\mathrm{S}}^{\mathrm{m}}\left(\Lambda^{2}\right), \mathrm{x}_{0}\right)
$$

PROOF. See $[8$, p. 47].

PROPOSITION 4.2. We have

$$
a_{\gamma}^{P}={ }^{t}\left(a_{\gamma, 1}^{P}, \ldots, a_{\gamma, m+1}^{P}\right)+\left(I-M_{\gamma}^{m}\right) m ! P_{m}^{-1}{ }^{t} c,
$$

where

$$
a_{\gamma, i}^{P}=\frac{(-1)^{m}{ }_{m}}{\left(\begin{array}{c}
m \\
m-i+1
\end{array}\right)} \int_{\gamma} \frac{\hat{A}_{m+2-i, x}}{W_{S}{ }^{m}\left(\Lambda^{2}\right), x} d x, \quad i=1, \ldots, m+1,
$$

and $c=\left(c_{1}, \ldots, c_{m+1}\right) \in C^{m+1}$ is a constant row vector.

PROOF. See [8, Proposition 3 bis. 13 and 14].

PROPOSITION 4.3.

$$
\mathrm{w}_{\mathrm{S}} \mathrm{m}\left(\Lambda^{2}\right), \mathrm{x}=1 ! 2 ! \ldots \mathrm{m} ! \mathrm{w}^{\mathrm{m}(\mathrm{m}+1) / 2} .
$$

PROOF. See [8, Theorem 3 bis. 5]. 
PROPOSITION 4.4 .

$$
\widehat{A}_{i, x}=1 ! 2 ! \ldots(m-1) !\left(\begin{array}{c}
m \\
i-1
\end{array}\right) \underset{\omega^{m}(m-1) / 2}{\Lambda^{2}, x} \omega_{1}^{i-1} \omega_{2}^{m-i+1}
$$

PROOF. See [8, Theorem 3 bis. 15].

PROPOSITION 4.5. Let $g(z)$ be the mixed automorphic form of type $(2, \mathrm{~m})$ associated to the differential equation

$$
S^{m}\left(\Lambda^{2}\right) f=Z, \quad Z \in \operatorname{LE}\left(S^{m}\left(\Lambda^{2}\right)\right) .
$$

Then we have

$$
g(z)=(-1)^{m+2} \frac{w_{2}(z)^{m} Z(z)}{W_{\Lambda^{2}, x}(z)^{m}}\left(\frac{d x}{d z}\right) .
$$

PROOF. We have

$$
\frac{d^{m} F}{d \omega^{m}}=m !\left(\int_{z_{0}}^{z}(-1)^{m+2} \frac{\hat{A}_{1, x^{Z}}}{W_{S^{m}}\left(\Lambda^{2}\right), x}\left(\frac{d x}{d z}\right) d z+c_{1}\right),
$$

so that

$$
g(z)=\frac{d}{d z}\left(\frac{d^{m} F}{d \omega^{m}}\right)=(-1)^{m+2} m ! \frac{\widehat{A}_{1, x^{Z}}}{W_{S^{m}}\left(\Lambda^{2}\right), x}\left(\frac{d x}{d z}\right) .
$$

By Proposition 4.3 and Proposition 4.4, we have

$$
\begin{gathered}
\mathrm{W}_{\mathrm{S}}{ }^{\mathrm{m}}\left(\Lambda^{2}\right), \mathrm{x} \\
(\mathrm{z})=1 ! 2 ! \ldots \mathrm{m} !\left(\mathrm{W}_{\Lambda^{2}, \mathrm{x}}(\mathrm{z})\right)^{\mathrm{m}(\mathrm{m}+1) / 2}, \\
\hat{\mathrm{A}}_{1, \mathrm{x}}=1 ! 2 ! \ldots(\mathrm{m}-1) ! \mathrm{W}^{\mathrm{m}(\mathrm{m}-1) / 2}{ }_{\Lambda^{2}, \mathrm{~m}}^{\mathrm{m}} .
\end{gathered}
$$

Thus it follows that

$$
g(z)=(-1)^{m+2} \frac{\omega_{2}(z)^{m} Z(z)}{{ }^{w} \Lambda^{2}, x^{(z)}}\left(\frac{d x}{d z}\right) .
$$

THEOREM 4.6. Let $g(z)$ be the mixed automorphic form of type $(2, m)$ associated to the differential equation

$$
\mathrm{s}^{\mathrm{m}}\left(\Lambda^{2}\right) \mathrm{f}=\mathrm{Z}, \quad \mathrm{Z} \in \operatorname{LE}\left(\mathrm{S}^{\mathrm{m}}\left(\Lambda^{2}\right)\right) .
$$

Then the associated parabolic cocycle $\left\{a_{\gamma}^{P}\right\}$ is determined by the formula

$$
a_{\gamma}^{P}=\left(a_{\gamma, 1}^{P}, \ldots, a_{\gamma, m+1}^{P}\right)+\left(I-M_{\gamma}^{m}\right) m ! P_{m}^{-1} t_{c}
$$

for $i=1, \ldots, \mathrm{m}+1$.

PROOF. By Proposition 4.2, it suffices to show that

$$
\frac{(-1)^{m} m !}{\left(\begin{array}{c}
m \\
m-i+1
\end{array}\right)} \int_{\gamma} \frac{\hat{A} z}{\mathrm{w}_{S^{m}}\left(\Lambda^{2}\right), x} d x=\int_{z_{0}}^{\gamma z} g(z) \omega(z)^{i-1} d z .
$$

Using Proposition 4.2 and Proposition 4.4, we have 


$$
\int_{\gamma} \frac{\hat{A}_{i, x^{Z}}}{W_{S} m\left(\Lambda^{2}\right), x} d x=\frac{(-1)^{m}}{m !}\left(\begin{array}{c}
m \\
i-1
\end{array}\right) \int_{\gamma}(-1)^{m+2} \frac{\omega_{2}^{m} Z}{w^{m+1}} \omega^{i-1} d x .
$$

By Proposition 4.5, we have

$$
(-1)^{\mathrm{m}+2} \frac{\omega_{2}^{\mathrm{m}} z}{W^{\mathrm{m}+1}} \mathrm{dx}=\mathrm{g}(\mathrm{z}) \mathrm{d} \mathrm{z}
$$

from which the theorem follows.

This result should be compared to [8, Theorem 3 bis. 17].

\section{REFERENCES}

1. Eichler, M. Eine Verallgemeinerung der Abelschen Integrale, Math. Z. 67 (1957), 267-293.

2. Hunt, B. and Meyer, W. Mixed automorphic forms and invariants of elliptic surfaces, Math. Ann. 271 (1985), 53-80.

3. Lee, M. H. Mixed automorphic forms and holomorphic forms on elliptic varieties, Pac. J. Math. 132 (1988), 363-370.

4. Lee, M. H. Mixed cusp forms and Hodge structures, to appear.

5. Lee, M. H. Periods of mixed cusp forms, to appear.

6. Shimura, G. Sur les intégrales attachés aux formes automorphes, J. Math. Soc. Japan 11 (1959), 291-311.

7. Šokurov, V. Holomorphic differential forms of higher degree on Kuga's modular varieties, Math. USSR Sb. 30 (1976), 119-142.

8. Stiller, P. Special values of Dirichlet series, monodromy, and the periods of automorphic forms, Mem. Amer. Math. Soc. No.299 (1984). 


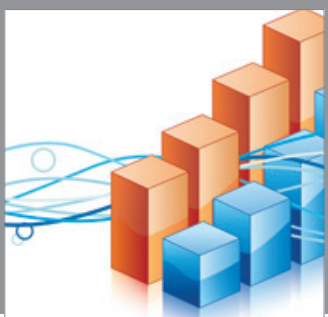

Advances in

Operations Research

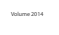

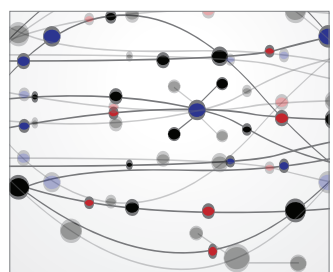

\section{The Scientific} World Journal
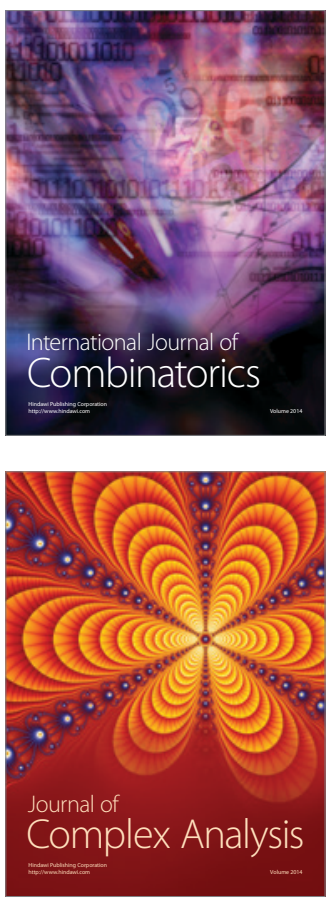

International Journal of

Mathematics and

Mathematical

Sciences
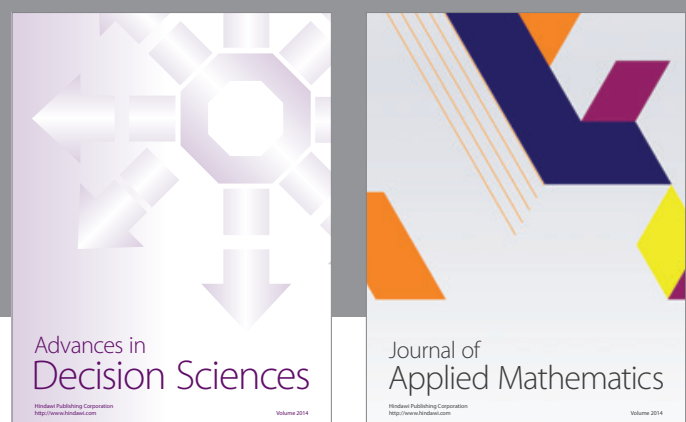

Journal of

Applied Mathematics
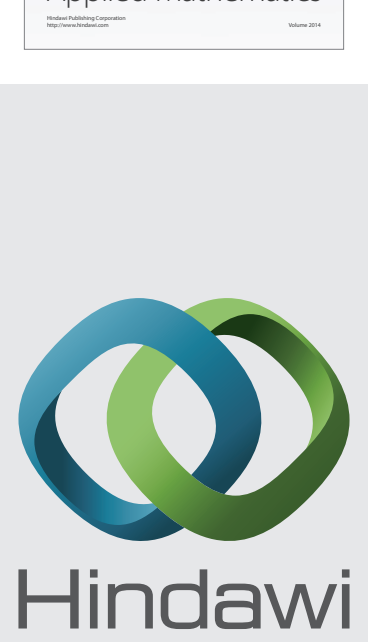

Submit your manuscripts at http://www.hindawi.com
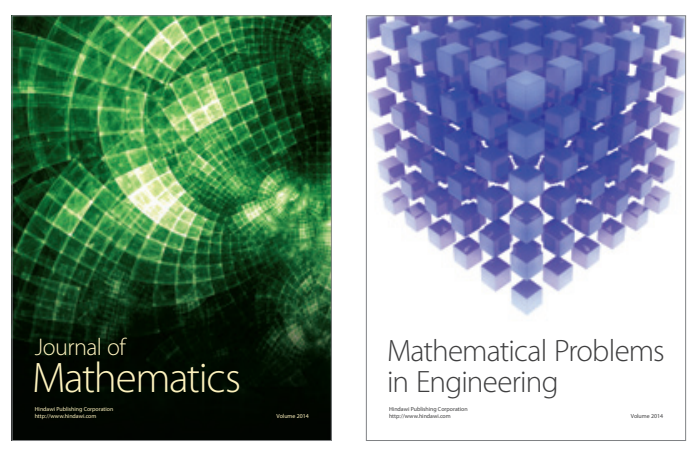

Mathematical Problems in Engineering
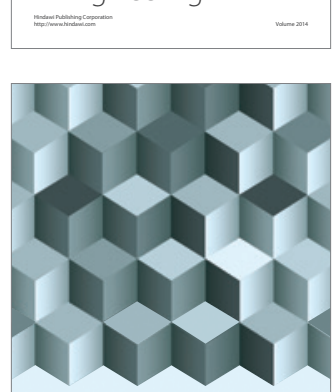

Journal of

Function Spaces
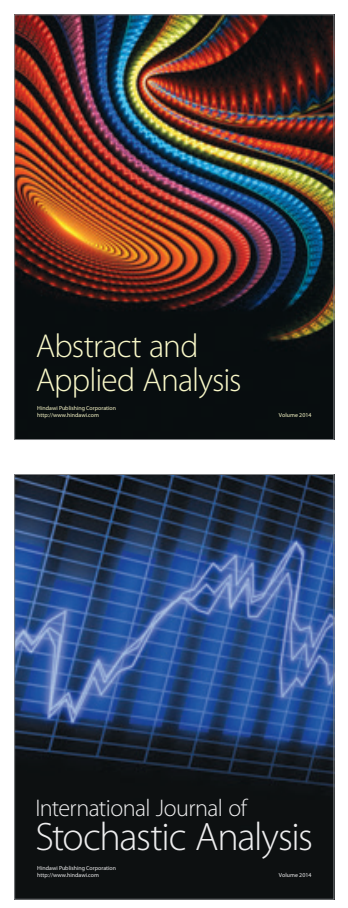

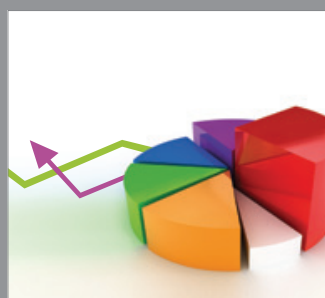

ournal of

Probability and Statistics

Promensencen
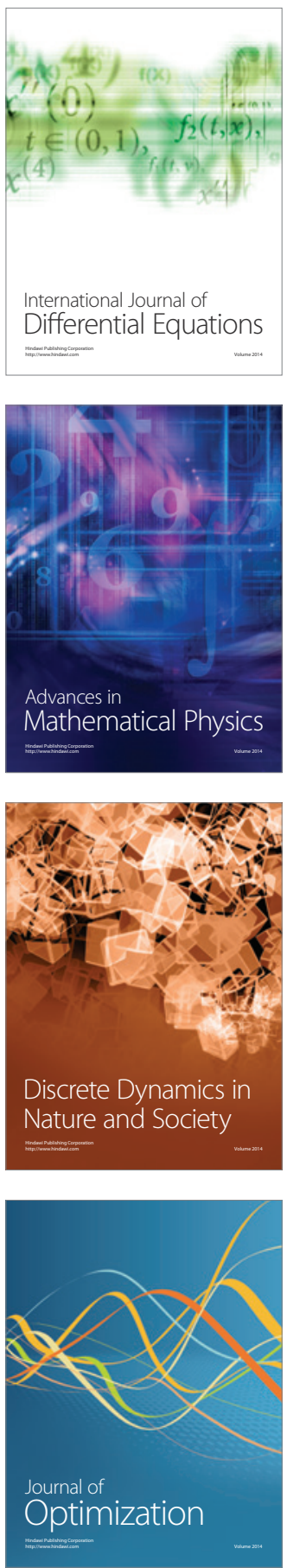